\title{
Stage III N2 non-small cell lung cancer treatment: decision-making among surgeons and radiation oncologists
}

\author{
Markus Glatzer ${ }^{1}$, Pawel Leskow ${ }^{2}$, Francesca Caparrotti ${ }^{3}$, Olgun Elicin ${ }^{4}$, Markus Furrer ${ }^{5}$, \\ Franco Gambazzi ${ }^{6}$, André Dutly ${ }^{2}$, Hans Gelpke $^{7}$, Matthias Guckenberger ${ }^{8}$, Jürg Heuberger', \\ Rolf Inderbitzi $^{10}$, Stefano Cafarotti ${ }^{10}$, Wolfram Karenovics ${ }^{11}$, Peter Kestenholz ${ }^{12}$, Gregor Jan Kocher ${ }^{13}$, \\ Peter Kraxner ${ }^{14,15}$, Thorsten Krueger ${ }^{16}$, Francesco Martucci ${ }^{17}$, Christoph Oehler ${ }^{18}$, Mahmut Ozsahin ${ }^{19}$, \\ Alexandros Papachristofilou ${ }^{20}$, Dirk Wagnetz ${ }^{21}$, Kathrin Zaugg ${ }^{22}$, Daniel Zwahlen ${ }^{18}$, Isabelle Opitz ${ }^{23}$, \\ Paul Martin Putora ${ }^{1,24}$
}

${ }^{1}$ Department of Radiation Oncology, Kantonsspital St. Gallen, St. Gallen, Switzerland; ${ }^{2}$ Department of Thoracic Surgery, Kantonsspital St. Gallen, St. Gallen, Switzerland; ${ }^{3}$ Department of Radiation Oncology, University Hospital Geneva, Geneva, Switzerland; ${ }^{4}$ Department of Radiation Oncology, Inselspital, Bern University Hospital, Bern, Switzerland; ${ }^{5}$ Department of Vascular and Thoracic Surgery, Kantonsspital Chur, Chur, Switzerland; ${ }^{6}$ Department of Thoracic Surgery, Kantonsspital Aarau, Aarau, Switzerland; ${ }^{7}$ Department of Thoracic Surgery, Kantonsspital Winterthur, Winterthur, Switzerland; ${ }^{8}$ Department of Radiation Oncology, University Hospital Zurich, University of Zurich, Zurich, Switzerland; ${ }^{9}$ Department of Radiation Oncology, Kantonsspital Aarau, Aarau, Switzerland; ${ }^{10}$ Department of Thoracic Surgery, Ente Ospedaliero Cantonale, Belinzona, Switzerland; ${ }^{11}$ Department of Thoracic Surgery, University Hospital Geneva, Geneva, Switzerland; ${ }^{12}$ Department of Thoracic Surgery, Kantonsspital Luzern, Luzern, Switzerland; ${ }^{13}$ Department of Thoracic Surgery, Inselspital, Bern University Hospital, University of Bern, Switzerland; ${ }^{14}$ Department of Radiation Oncology, Kantonsspital Luzern, Luzern, Switzerland; ${ }^{15}$ Department of Radiation Oncology, Kantonsspital Chur, Chur, Switzerland; ${ }^{16}$ Department of Thoracic Surgery, University Hospital Lausanne, Lausanne, Switzerland; ${ }^{17}$ Radiation Oncology Clinic, Oncology Institute of Southern Switzerland, Bellinzona-Lugano, Switzerland; ${ }^{18}$ Department of Radiation Oncology, Kantonsspital Winterthur, Winterthur, Switzerland; ${ }^{19}$ Department of Radiation Oncology, Centre Hospitalier Universitaire Vaudois, Lausanne, Switzerland; ${ }^{20}$ Department of Radiation Oncology, University Hospital Basel, Basel, Switzerland; ${ }^{21}$ Department of Visceral-Vascular and Thoracic Surgery, City Hospital Waid and Triemli, Zurich, Switzerland; ${ }^{22}$ Department of Radiation Oncology, City Hospital Waid and Triemli, Zurich, Switzerland; ${ }^{23}$ Department of Thoracic Surgery, University Hospital Zurich, Zurich, Switzerland; ${ }^{24}$ Department of Radiation Oncology, University of Bern, Bern, Switzerland Contributions: (I) Conception and design: PM Putora; (II) Administrative support: M Glatzer; (III) Provision of study materials or patients: P Leskow, F Caparrotti, O Elicin, M Furrer, F Gambazzi, A Dutly, H Gelpke, M Guckenberger, J Heuberger, R Inderbitzi, S Cafarotti, W Karenovics, P Kestenholz, GJ Kocher, P Kraxner, T Krüger, F Martucci, C Oehler, M Ozsahin, A Papachristofilou, D Wagnetz, K Zaugg, D Zwahlen, I Opitz, PM Putora; (IV) Collection and assembly of data: PM Putora, P Leskow; (V) Data analysis and interpretation: PM Putora, M Glatzer, P Leskow; (VI) Manuscript writing: All authors; (VII) Final approval of manuscript: All authors.

Correspondence to: Markus Glatzer. Department of Radiation Oncology, Kantonsspital St. Gallen, Rorschacher Strasse 95 , 9007 St. Gallen, Switzerland. Email: markus.glatzer@kssg.ch.

Background: Stage III N2 non-small cell lung cancer (NSCLC) is a very heterogeneous disease associated with a poor prognosis. A number of therapeutic options are available for patients with Stage III N2 NSCLC, including surgery [with neoadjuvant or adjuvant chemotherapy (CTx)/neoadjuvant chemoradiotherapy (CRT)] or CRT potentially followed by adjuvant immunotherapy. We have no clear evidence demonstrating a significant survival benefit for either of these approaches, the selection between treatments is not always straightforward and can come down to physician and patient preference. The very heterogeneous definition of resectability of N2 disease makes the decision-making process even more complex.

Methods: We evaluated the treatment strategies for preoperatively diagnosed stage III cN2 NSCLC among Swiss thoracic surgeons and radiation oncologists. Treatment strategies were converted into decision trees and analysed for consensus and discrepancies. We analysed factors relevant to decision-making within these recommendations.

Results: For resectable "non-bulky" mediastinal lymph node involvement, there was a trend towards surgery. Numerous participants recommend a surgical approach outside existing guidelines as long as 
the disease was resectable, even in multilevel N2. With increasing extent of mediastinal nodal disease, multimodal treatment based on radiotherapy was more common.

Conclusions: Both, surgery- or radiotherapy-based treatment regimens are feasible options in the management of Stage III N2 NSCLC. The different opinions reflected in the results of this manuscript reinforce the importance of a multidisciplinary setting and the importance of shared decision-making with the patient.

Keywords: Non-small cell lung cancer (NSCLC); stage III N2 lung cancer; decision-making; surgery; radiotherapy

Submitted Nov 24, 2020. Accepted for publication Apr 14, 2021.

doi: $10.21037 /$ tlcr-20-1210

View this article at: http://dx.doi.org/10.21037/tlcr-20-1210

\section{Introduction}

Stage III non-small cell lung cancer (NSCLC) with subcarinal and/or ipsilateral mediastinal lymphatic spread (N2, TNM UICC $8^{\text {th }}$ edition) may be treated with curative intent. However, treatment outcome remains poor with 15 $50 \% 5$-year overall survival (OS) rates (1-4). The optimal treatment for potentially resectable N2 NSCLC patients is unclear due to the heterogeneity of the disease, a lack of a universal definition of resectability, and comparable outcomes with different treatment approaches. There is a variety of factors influencing the availability and suitability of various treatments (5) and a large number of clinical decision criteria used in daily decision-making (6). The two main multimodal treatment options for NSCLC N2 disease are surgery (with neoadjuvant chemotherapy (CTx)/ chemoradiotherapy (CRT) or combined with adjuvant CTx) or CRT [with or without adjuvant immunotherapy (7)].

Both treatment options may be available for several decision criteria combinations, especially since there is no randomised evidence suggesting a clear survival benefit with one approach over another $(4,8,9)$. This is reflected in international guidelines where radiotherapy and surgery are both options for selected stage III N2 NSCLC, especially in cases with limited lymph node involvement $(10,11)$. In such a setting, patient preference becomes even more relevant and the focus may shift away from OS towards side effects, quality of life and logistical issues (e.g., hospital stay for surgery or receiving daily outpatient treatments for several weeks with radiotherapy).

In daily clinical routine, there are scenarios where multiple treatment options are available. It is important to recognize these situations for an optimal shared decisionmaking approach. The aim of this work was to identify which disease characteristics are applied in current clinical practice among Swiss thoracic oncology centres for stage III N2 NSCLC and how they impact decision-making in clinical routine.

\section{Methods}

All Swiss centres providing both departments for thoracic surgery and radiation oncology with an established training program were asked to participate in this study. The initial search for qualifying centres was performed in October 2018 according to the list of the Swiss Institute for Medical Education (SIWF). Twelve centres were identified. The head of each radiation oncology and thoracic surgery department was asked to describe her or his practice in preoperatively diagnosed stage III N2 NSCLC. In some instances, a senior physician specialized in lung cancer represented the department. The collected answers from 11 surgeons and 12 radiation oncologists were anonymously analysed, one expert declined to participate. All university hospitals as well as most Swiss cantonal hospitals were represented.

All participating centres were asked to provide their recommendations and decision criteria for the management of preoperatively diagnosed stage III N2 NSCLC. The recommendations were reduced to the options of: a definitive surgical approach (with neoadjuvant or adjuvant CTx/CRT), definitive CRT approach or "patient preference" (no specific recommendation for either RT or surgery; both being options). Decision criteria collected were converted into decision trees, analysed and standardized if their meaning was not changed as described previously (12-14). For better comparison, universal criteria 


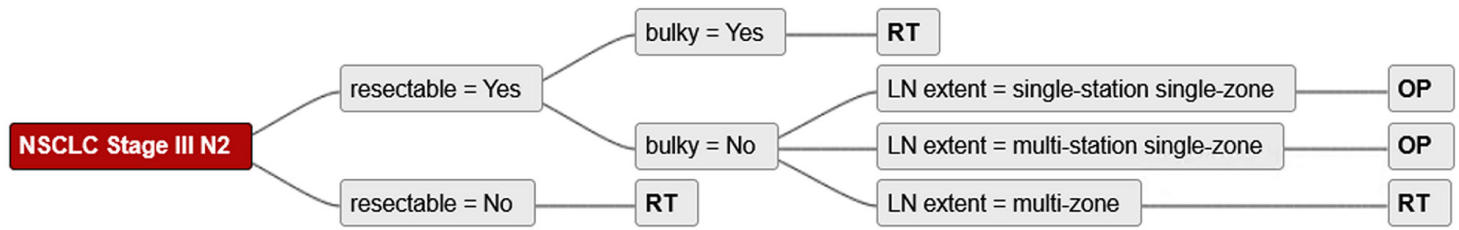

Figure 1 Example of the simplified decision tree. LN, lymph nodes; OP, surgery; RT, radiotherapy.

such as "ability to give informed consent" were not included in this analysis. In several instances, trial participation was a recommended option. This was excluded from the decision tree analysis, as trials are considered experimental and potentially not applicable to the routine clinical setting.

The resulting decision criteria included: resectability (potentially resectable or not), lymph node (LN) volume (bulky, non-bulky), and LN extent (single station, multi station and single zone, multi-zone). For the purpose of this analysis, "resectability" included the possibility of surgical tumour removal with an R0 margin based on preoperative imaging, as well as the required physical fitness for radical operative treatment.

The extent of mediastinal lymph node involvement was categorized as: (I) single station and therefore single zone; (II) multi-station but limited to single zone; and (III) multi-zone. This was in-line with the descriptions received from individual participants and was based on a previous analysis of international guidelines (11). In the British Thoracic Society (BTS) (15) and Irish guidelines (16) lymph nodes are grouped by zones according to the IASLC lymph node map (AJCC $7^{\text {th }}$ edition), whereas most others, e.g., ESMO $(10,17)$ or German (18) guidelines used nodal stations.

The descriptions used to characterise mediastinal lymph nodes varies among guidelines (11). Non-bulky lymph nodes are mostly described as lymph nodes with a diameter of less than $3 \mathrm{~cm}$ short-axis diameter, easily measurable and free of major mediastinal structures including the trachea and great vessels, or low volume lymph nodes $(15,19-22)$. Many experts described bulkiness or size of lymph nodes, while no specific cut-off in $\mathrm{cm}$ was defined, these criteria were simplified and represented as "bulky vs. non-bulky".

As the treatment of stage III N2 NSCLC is multimodal (11,23-26), the reduction to radiotherapy or surgerybased treatments represents a significant simplification. For multimodal treatment based on a surgical approach, no distinction was made between neoadjuvant and adjuvant chemotherapy $(19,20)$.

\section{Statistical analysis}

The resulting decision trees were analysed for consensus and discrepancies with the objective consensus methodology $(12,27,28)$. Majorities were calculated by dividing the most common recommendation for any specific situation by the number of participants.

\section{Results}

Twenty-three management strategies with recommendations for all unified decision criteria were identified and converted into decision trees. An example of a single decision tree is shown in Figure 1.

The three major decision criteria that affected treatment decisions were resectability, bulkiness of lymph nodes and lymph node extent. The three simplified treatment options included surgery, radiotherapy, and patient preference.

For non-resectable cases, radiotherapy-based treatments were recommended by all experts.

For patients with non-bulky lymph nodes a majority recommended surgery. In case of single-station non-bulky disease, $91 \%$ of experts recommended surgery as the single most common recommendation. In case of multi-station, but single-zone disease, still $83 \%$ recommended surgery as the single most common recommendation. For multi-zone non-bulky disease, a slight majority (65\%) for surgery was identified with 7 experts (30\%) stating radiotherapy and one (4\%) stating patient preference as their recommendation (Figure 2).

In bulky disease, the majority (74-83\%, dependant on LN extent) recommended radiotherapy; however, for example in single-station disease, five experts (22\%) recommended surgery and one (4\%) recommended patient preference. In cases where surgery was recommended for bulky disease comment was added by the experts that a neoadjuvant approach (with chemotherapy or radio-chemotherapy) was 


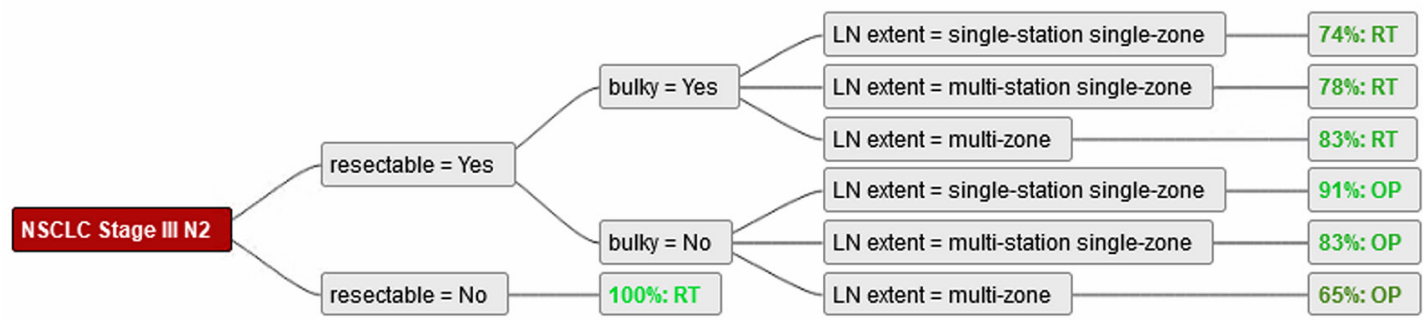

Figure 2 demonstrates the overall majority recommendations for each parameter combination. LN, lymph nodes; OP, surgery; RT, radiotherapy.

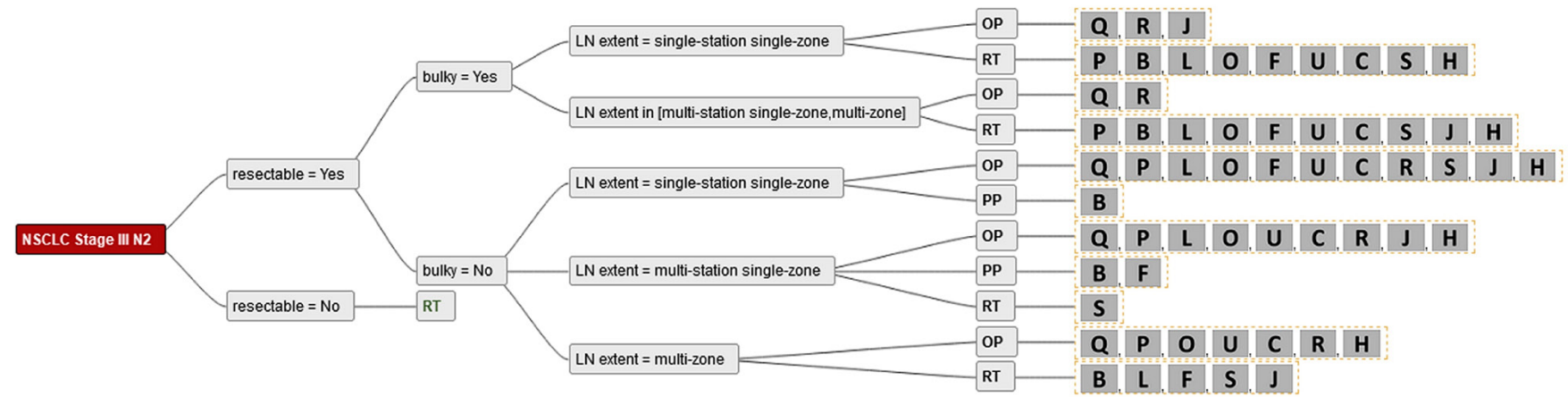

Figure 3 Demonstrates the answers from the radiation oncologists for all combinations of simplified criteria. Single alphabetic letters: participating sites. LN, lymph nodes; OP, surgery; PP, patient's preference; RT, radiotherapy.

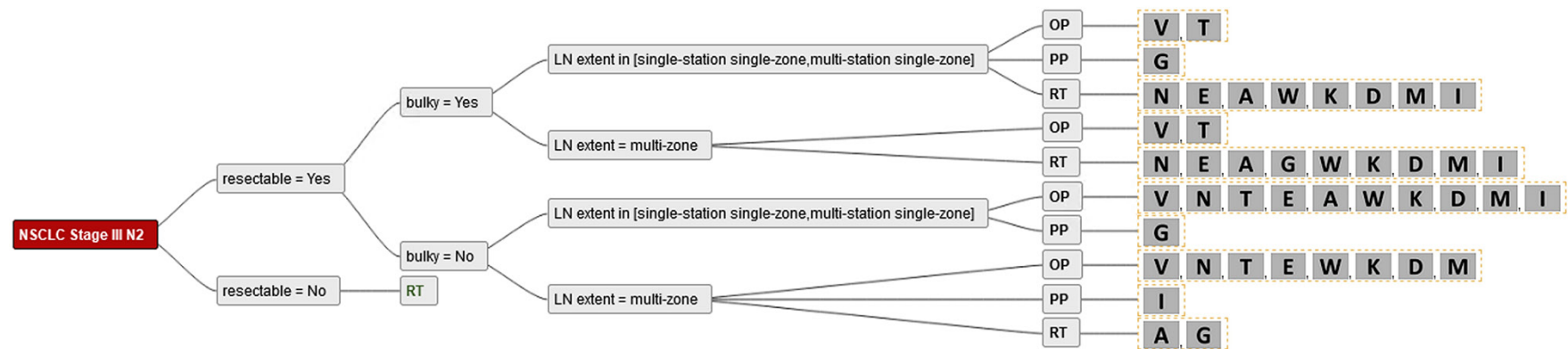

Figure 4 Demonstrates the answers from surgeons for all combinations of simplified criteria. Single alphabetic letters: participating sites. LN, lymph nodes; OP, surgery; PP, patient's preference; RT, radiotherapy.

recommended and that the surgery would be performed only if a good response was observed (Figures 2-4).

Patient preference as a recommendation only occurred in 4 settings, this included 2 radiation oncologists and two surgeons in various situations (Figures 3,4). A trend towards radiotherapy among radiation oncologists and surgery among thoracic surgeons was observed (Figures 2,3). Seventy-three percent of participating surgeons would recommend a surgical approach and $18 \%$ would recommend
CRT in resectable, non-bulky, multizone stage III N2 NSCLC, whereas $42 \%$ of radiation oncologists would recommend CRT for the same condition and surgery in $58 \%$.

\section{Discussion}

Our decision-making analysis revealed surgery-based approaches as the most commonly recommended treatments for resectable stage III N2 NSCLC, with a 
significant proportion of recommendations considering radiotherapy as an alternative. Overall, there was a stronger preference for surgical approaches the smaller the lymphnode extent was (Figure 4). For non-resectable disease, there was a consensus for radiotherapy as well as a clear majority for radiotherapy in bulky disease.

Resectability is a prerequisite for surgery in stage III N2 NSCLC. Preoperative surgical evaluation can be challenging and dependent on the individual surgeon's expertise. Universally agreed-upon criteria to guarantee the resectability of disease are lacking. Additionally preoperative and postoperative findings may diverge (29). The difficulty in standardization lies in the surgeon's individual judgement on being able to achieve a clear resection margin, the assessment of which may be particularly challenging in more centrally located tumours. The definition of an unresectable tumour in this analysis was left to the decision of multidisciplinary tumour boards and was highly variable among institutions.

Overall, with increasing volume or extent of N2 disease, there was an increasing trend towards therapy based on RT. There was no consensus on a unified cut off for nodal volume related to surgery. In patients with low volume (especially single station) mediastinal N2 disease surgery was the preferred recommendation. Some guidelines recommend surgery for N2 disease without additional risk factors (such as bulky disease or multi-station disease, e.g., ESMO) or mention both radiotherapy- or surgery-based options (e.g., NCCN) (10,30). In our analysis, $91 \%$ of participating sites recommended a multimodal treatment based on surgery for single station (single-zone) non-bulky stage III N2 NSCLC, in two cases (9\%) patients' preference was stated (offering CRT as an equivalent option in this setting). The recommendation rate for surgery-based approaches decreased with increasing mediastinal lymph node involvement, nevertheless $83 \%$ of participating surgeons as well as $75 \%$ radiation oncologists still recommended surgery as an option for multi-station, but single zone stage III N2 NSCLC. With multi-zone involvement, a slight preference for surgery could be shown (65\%) even though this is not inline with most guidelines (11). In this setting, a discrepancy between specialties was visible with $42 \%$ of radiation oncologists recommending radiotherapy, while only $18 \%$ of surgeons would not recommend surgery in NSCLC with multi-zone non-bulky LN involvement. A minor limitation of our simplification is that the primary multi-station nodal involvement described in a guideline using nodal stations as a descriptor could fall within a single zone or multiple zones (e.g., stations $2 \& 4$ would be a single zone, stations 4 \& 7 would be multi-zone)

A lack of a clear definition of bulky lymph nodes leads to potential bias. Some consider $3 \mathrm{~cm}$ as the critical diameter for bulky definition (11), some authors consider bulky N2 disease as lymph nodes already greater than $2.5 \mathrm{~cm}$ in short axis (31). The preference of radiotherapy-based approaches increases when mediastinal lymph nodes present as bulky. For all settings with bulky lymph nodes, radiotherapy-based approaches were the most recommended treatment. The rate for recommending radiotherapy-based approaches in bulky disease increased from $74 \%$ for single-station to $78 \%$ for multi-station single zone and to $83 \%$ for multi-zone bulky lymph node involvement. This trend is clearly in line with guidelines advising against surgery in the presence of bulky lymph nodes. The individual experts recommending surgical approaches in this setting also recommended neoadjuvant chemotherapy or radiotherapy with the aim of downstaging and considered a good response as well as an expectation of an R0 resection to be prerequisites for surgery. However, with a neoadjuvant treatment approach the risk of mediastinal soft tissue fibrosis may increase and may complicate subsequent hilar and mediastinal dissections (31,32).

A meta-analysis including randomized studies comparing definitive radiochemotherapy versus surgery in stage III NSCLC (4) found no significant difference between bimodal treatments (surgery $v s$. radiotherapy based) related to overall survival. There was heterogeneity across studies at two years, resulting from the rate of pneumonectomies performed. The increased mortality following pneumonectomies however was not confirmed in other trials $(3,33,34)$. Another previous meta-analysis by McElnay et al. (9) compared CRT and surgery versus definitive CRT. It combined data from the Intergroup 0139 trial (2) and a study from Sorensen et al. (35) comparing induction CTx followed by RT versus induction CTx followed by surgery followed by RT. Both studies were weighted equally in the analysis. The pooled HR for death in the surgery group was 0.87 (95\% CI: 0.75 to $1.01 ; \mathrm{P}=0.068)$ with no statistical evidence of heterogeneity $(\mathrm{P}=0.976)$. Trimodal therapy was associated with a trend towards improved survival compared to bimodal treatments. However, comparisons between surgery and RT are complicated by early perioperative mortality of surgical patients with potential advantages in survival later on (36).

The 2019 NICE guidelines cover the management of NSCLC. For potentially resectable stage III N2 NSCLC the guideline specifically recommended trimodality treatment for patients fit enough for multi-modality treatment (induction CRT followed by surgery) (37). This is a highly 
significant change from previous NICE recommendations and a significant change in UK practice where only $1.1 \%$ of $\mathrm{N} 2$ patients currently receive trimodality treatment (38). The meta-analysis of the NICE guideline group compared CRT followed by surgery (CRS), CRT, and chemotherapy and surgery (CS). A statistically clear survival benefit could not demonstrate between treatment regimens, but there was a trend towards improved survival with CRS (in line with the trends from other meta-analyses). The PFS at 4 \& 5 years (high quality evidence) was improved in the CRS group with an average of 4.5 month compared to CRT/CS. There was less grade 3+ adverse events with CRS than with $\mathrm{CR} / \mathrm{CS}$. The guideline also undertook cost-effectiveness modelling concluding that CRT is more cost effective than CS [Incremental cost-effectiveness ratio (ICER) £53,000/ quality-adjusted life year (QALY)] and CRS is more cost effective than CRT (ICER \&17,800/QALY). Even though the guideline recommended trimodality treatment, such practice would require careful and considered implementation within agreed protocols through high volume centres where outcomes are monitored closely. In our analysis only 2 of 23 experts recommended trimodality treatment in their clinical routine.

Overall, the evidence in this setting has several severe limitations. A number of key trials (Intergroup/EORTC) date from a time before routine ${ }^{18} \mathrm{FDG}-\mathrm{PET} / \mathrm{CT}$, endoscopic nodal staging and advances in surgical and RT techniques are not accounted for. Some trials, such as the ESPATUE trial (3) had a heterogeneous study population (e.g., 1/3 of patients with T4 N0/1) further hampering definitive conclusions on the management of $\mathrm{N} 2$ disease.

Over the past years, immunotherapy has changed the landscape for patients with stage III NSCLC. Also, in the management of stage III N2 NSCLC this approach is part of ongoing trials (NCT04202809, NCT04287894 and NCT04245514). The implementation of immunotherapy in international treatment guidelines is progressing, however has not directly affected the decision between surgical and RT-based approaches yet. In unresectable stage III N2 NSCLC treated with CRT, a significantly longer progression free survival (PFS) and OS was reached with the addition of maintenance durvalumab and this has become a recommended strategy in guidelines $(7,39)$.

There are also considerations of using immunotherapy in the neoadjuvant setting. A study by Bott et al. (40) demonstrated a major pathological response in $45 \%$ of patients treated with nivolumab upfront of surgery, this trial enrolled NSCLC stage I-IIIA. However, more than a half of the minimally invasive-intended operations were converted to thoracotomy due to hilar inflammation and fibrosis. While there was no operative mortality, perioperative morbidity was present and mostly due to atrial arrhythmia. Similar results were published by Forde et al., with major pathological response rates on histologic examination of around $45 \%$. Interestingly only two patients had a radiologic partial response (41). It was due to postulated immune-cell infiltration into the tumour, rather than true tumour mass. The phase II trial by Sacha Rothschild et al. (42) also demonstrated a high response rate and a very encouraging 1 -year event-free survival by addition of perioperative durvalumab to standard of care cisplatin/docetaxel. To define the role of neoadjuvant immunotherapy long-term follow-up of currently recruiting phase 3 studies such as NCT02998528 need to be evaluated. The evolution of future treatment strategies involving immunotherapy need further prospective studies to answer the question whether CRT with Immunotherapy is superior to multimodal treatment based on surgery in potentially resectable stage III N2 NSCLC.

As shared decision-making is a complex process with various potential pitfalls (43), patient preference will become increasingly helpful if more than one treatment regimen can be offered with comparable outcome $(5,44,45)$. Patient preference was a primary recommendation in selected settings, mentioned only by a limited number of experts (these represented both specialities). Patient preference may be challenging in clinical practice as a multitude of emotions and biases influence shared decisionmaking $(43,46)$. This process is even further complicated by the limited availability of patient-relevant informationsuch as detailed information from side effects from clinical trials in this setting (47). Potentially a third party acting as an intermediary between the specialists may be of value in providing an un-biased view (e.g., pneumologist or general practitioner).

The results of this analysis are based on the input of specific individual experts in Switzerland, yet it is improbable that the trends observed would be significantly different if other experts from Switzerland would have been selected. A specialty bias was visible with trends towards surgery among surgeons and radiotherapy among radiation oncologists, this only reinforces the need for such analyses to be performed in a multidisciplinary setting.

\section{Conclusions}


Our analysis showed that in patients with low mediastinal lymph node involvement and resectable tumours a multimodal approach based on surgical resection is the preferred treatment practice in Switzerland, whereas with increasing mediastinal lymph node involvement, multimodal therapy based on radiation is being considered more frequently. Compared to recommended treatment pathways within international guidelines, surgery-based treatment for stage III N2 NSCLC in Switzerland is offered more extensively. Definitive evidence for the optimal treatment of resectable stage III N2 NSCLC remains elusive. The variability of recommendations among the participating sites may have multiple potential reasons. These may include local traditions (e.g., through the participation in the Swiss SAKK 16/00 trial investigation neoadjuvant radiochemotherapy in Stage IIIA N2 NSCLC), local technical equipment (ECMO), level of individual surgical or radiotherapy experience, incomplete definition of decision criteria (e.g., only broadly specified lymph node involvement, lacking definition of resectability) as well as different guideline used for development of local recommendation of the interdisciplinary tumour board.

The different opinions reflected in the results of this manuscript reinforce the importance of a multidisciplinary setting and the importance of shared decision-making with the patient.

\section{Acknowledgments}

Funding: We thank AstraZeneca for an educational grant that enabled this project.

\section{Footnote}

Provenance and Peer Review: This article was commissioned by the guest editors (Jacek Jassem and Rafal Dziadziuszko) for the series "Radiotherapy in thoracic malignancies" published in Translational Lung Cancer Research. The article has undergone external peer review.

Data Sharing Statement: Available at http://dx.doi. org/10.21037/tlcr-20-1210

Peer Review File: Available at http://dx.doi.org/10.21037/ tlcr-20-1210

Conflicts of Interest: All authors have completed the ICMJE uniform disclosure form (available at http://dx.doi. org/10.21037/tlcr-20-1210). The series "Radiotherapy in thoracic malignancies" was commissioned by the editorial office without any funding or sponsorship. PMP received an educational grant from AstraZeneca (educational grant to the Institution); outside the submitted work, he also received research support and educational grants to the department from Celgene, Roche and Takeda. The authors have no other conflicts of interest to declare.

Ethical Statement: The authors are accountable for all aspects of the work in ensuring that questions related to the accuracy or integrity of any part of the work are appropriately investigated and resolved. Ethics approval was not needed as no patient data were used.

Open Access Statement: This is an Open Access article distributed in accordance with the Creative Commons Attribution-NonCommercial-NoDerivs 4.0 International License (CC BY-NC-ND 4.0), which permits the noncommercial replication and distribution of the article with the strict proviso that no changes or edits are made and the original work is properly cited (including links to both the formal publication through the relevant DOI and the license). See: https://creativecommons.org/licenses/by-nc-nd/4.0/.

\section{References}

1. van Meerbeeck JP, Kramer GW, Van Schil PE, et al. Randomized controlled trial of resection versus radiotherapy after induction chemotherapy in stage IIIA-N2 non-small-cell lung cancer. J Natl Cancer Inst 2007;99:442-50.

2. Albain KS, Swann RS, Rusch VW, et al. Radiotherapy plus chemotherapy with or without surgical resection for stage III non-small-cell lung cancer: a phase III randomised controlled trial. Lancet 2009;374:379-86.

3. Eberhardt WE, Pottgen C, Gauler TC, et al. Phase III Study of Surgery Versus Definitive Concurrent Chemoradiotherapy Boost in Patients With Resectable Stage IIIA(N2) and Selected IIIB Non-Small-Cell Lung Cancer After Induction Chemotherapy and Concurrent Chemoradiotherapy (ESPATUE). J Clin Oncol 2015;33:4194-201.

4. Pöttgen C, Eberhardt W, Stamatis G, et al. Definitive radiochemotherapy versus surgery within multimodality treatment in stage III non-small cell lung cancer (NSCLC)-a cumulative meta-analysis of the randomized evidence. Oncotarget 2017;8:41670. 
5. Panje CM, Glatzer M, Siren C, et al. Treatment Options in Oncology. JCO Clin Cancer Inform 2018;2:1-10.

6. Glatzer M, Panje CM, Siren C, et al. Decision Making Criteria in Oncology. Oncology 2020;98:370-8.

7. Antonia SJ, Villegas A, Daniel D, et al. Overall Survival with Durvalumab after Chemoradiotherapy in Stage III NSCLC. N Engl J Med 2018;379:2342-50.

8. Evison M, Clive A, Castle L, et al. Resectable Clinical N2 Non-Small Cell Lung Cancer; What Is the Optimal Treatment Strategy? An Update by the British Thoracic Society Lung Cancer Specialist Advisory Group. J Thorac Oncol 2017;12:1434-41.

9. McElnay PJ, Choong A, Jordan E, et al. Outcome of surgery versus radiotherapy after induction treatment in patients with N2 disease: systematic review and metaanalysis of randomised trials. Thorax 2015;70:764-8.

10. Postmus PE, Kerr KM, Oudkerk M, et al. Early and locally advanced non-small-cell lung cancer (NSCLC): ESMO Clinical Practice Guidelines for diagnosis, treatment and follow-up. Ann Oncol 2017;28:iv1-21.

11. Putora PM, Leskow P, McDonald F, et al. International guidelines on stage III N2 nonsmall cell lung cancer: surgery or radiotherapy? ERJ Open Res 2020;6:001592019.

12. Panje CM, Glatzer M, von Rappard J, et al. Applied Swarm-based medicine: collecting decision trees for patterns of algorithms analysis. BMC Med Res Methodol 2017;17:123.

13. Zumstein V, Betschart P, Abt D, et al. Surgical management of urolithiasis - a systematic analysis of available guidelines. BMC Urol 2018;18:25.

14. Hundsberger T, Schoser B, Leupold D, et al. Comparison of recent pivotal recommendations for the diagnosis and treatment of late-onset Pompe disease using diagnostic nodes - the Pompe disease burden scale. J Neurol 2019;266:2010-7.

15. Lim E, Baldwin D, Beckles M, et al. Guidelines on the radical management of patients with lung cancer. Thorax 2010;65 Suppl 3:iii1-27.

16. Health" Do. Diagnosis, staging and treatment of lung cancer (NCEC National Clinical Guideline No. 16). 2017. Available online: http://health.gov.ie/national-patientsafety-office/ncec/national-clinical-guidelines

17. Eberhardt WE, De Ruysscher D, Weder W, et al. 2nd ESMO Consensus Conference in Lung Cancer: locally advanced stage III non-small-cell lung cancer. Ann Oncol 2015;26:1573-88.

18. "Leitlinienprogramm Onkologie (Deutsche
Krebsgesellschaft DK, AWMF)". Prävention, Diagnostik, Therapie und Nachsorge des Lungenkarzinoms, Langversion 1.0. 2018. Available online: http://leitlinienprogramm-onkologie.de/ Lungenkarzinom.98.0.html

19. Ettinger DS, Aisner DL, Wood DE, et al. NCCN Guidelines Insights: Non-Small Cell Lung Cancer, Version 5.2018. J Natl Compr Canc Netw 2018;16:80721.

20. Ettinger DS, Wood DE, Aisner DL, et al. Nonsmall cell lung cancer, version 5.2017, NCCN clinical practice guidelines in oncology. Journal of the National Comprehensive Cancer Network 2017;15:504-35.

21. Zhi XY, Yu JM, Shi YK. Chinese guidelines on the diagnosis and treatment of primary lung cancer $(2015$ version). Cancer 2015;121 Suppl 17:3165-81.

22. Silvestri GA, Gonzalez AV, Jantz MA, et al. Methods for staging non-small cell lung cancer: Diagnosis and management of lung cancer, 3rd ed: American College of Chest Physicians evidence-based clinical practice guidelines. Chest 2013;143:e211S-50S.

23. Winton T, Livingston R, Johnson D, et al. Vinorelbine plus cisplatin vs. observation in resected non-small-cell lung cancer. N Engl J Med 2005;352:2589-97.

24. Horinouchi H. Role of multimodality therapy in cIIIA-N2 non-small cell lung cancer: perspective. Jpn J Clin Oncol 2016;46:1174-8.

25. Arriagada R, Bergman B, Dunant A, et al. Cisplatinbased adjuvant chemotherapy in patients with completely resected non-small-cell lung cancer. N Engl J Med 2004;350:351-60.

26. Massard G, Renaud S, Reeb J, et al. N2-IIIA nonsmall cell lung cancer: a plea for surgery! J Thorac Dis 2016;8:S849-54.

27. Putora PM, Panje CM, Papachristofilou A, et al. Objective consensus from decision trees. Radiat Oncol 2014;9:270.

28. Putora PM, Glatzer M, Belderbos J, et al. Prophylactic cranial irradiation in stage IV small cell lung cancer: Selection of patients amongst European IASLC and ESTRO experts. Radiother Oncol 2019;133:163-6.

29. Quint LE. Lung cancer: assessing resectability. Cancer Imaging 2003;4:15-8.

30. National Comprehensive Cancer Network. Non-Small Cell Lung Cancer (Version 8.2020). Available online: https://www.nccn.org/professionals/physician_gls/pdf/ nscl.pdf

31. De Leyn P, Dooms C, Kuzdzal J, et al. Revised ESTS guidelines for preoperative mediastinal lymph node staging 
for non-small-cell lung cancer. Eur J Cardiothorac Surg 2014:45:787-98.

32. Van Schil P, Hendriks J, De Maeseneer M, et al. Decision making about operability in non-small cell lung cancer. Acta Chir Belg 2007;107:495-9.

33. Pricopi C, Mordant P, Rivera C, et al. Postoperative morbidity and mortality after pneumonectomy: a 30 year experience of 2064 consecutive patients. Interact Cardiovasc Thorac Surg 2015;20:316-21.

34. Weder W, Collaud S, Eberhardt WE, et al. Pneumonectomy is a valuable treatment option after neoadjuvant therapy for stage III non-small-cell lung cancer. J Thorac Cardiovasc Surg 2010;139:1424-30.

35. Sorensen JB, Riska H, Ravn J, et al. Scandinavian phase III trial of neoadjuvant chemotherapy in NSCLC stages IB-IIIA/T3. J Clin Oncol 2005;23. doi: 10.1200/ jco.2005.23.16_suppl.7146

36. Rusthoven CG, Palma DA, Senan S, et al. The Head Start Effect: Will Acute and Delayed Postoperative Mortality Lead to Improved Survival with Stereotactic Body Radiation Therapy for Operable Stage I Non-Small-Cell Lung Cancer? J Clin Oncol 2017;35:1749-51.

37. Team NGU. National Institute for Health and Care Excellence: Clinical Guidelines. Lung cancer: diagnosis and management. London: National Institute for Health and Care Excellence (UK), 2019.

38. Adizie JB, Khakwani A, Beckett P, et al. Stage III nonsmall cell lung cancer management in England. Clin Oncol (R Coll Radiol) 2019;31:688-96.

39. Paz-Ares L, Spira A, Raben D, et al. Outcomes with durvalumab by tumour PD-L1 expression in unresectable,

Cite this article as: Glatzer M, Leskow P, Caparrotti F, Elicin O, Furrer M, Gambazzi F, Dutly A, Gelpke H, Guckenberger M, Heuberger J, Inderbitzi R, Cafarotti S, Karenovics W, Kestenholz P, Kocher GJ, Kraxner P, Krueger T, Martucci F, Oehler C, Ozsahin M, Papachristofilou A, Wagnetz D, Zaugg K, Zwahlen D, Opitz I, Putora PM. Stage III N2 non-small cell lung cancer treatment: decision-making among surgeons and radiation oncologists. Transl Lung Cancer Res 2021;10(4):19601968. doi: 10.21037/tlcr-20-1210
Stage III non-small-cell lung cancer in the PACIFIC trial. Ann Oncol 2020;31:798-806.

40. Bott MJ, Yang SC, Park BJ, et al. Initial results of pulmonary resection after neoadjuvant nivolumab in patients with resectable non-small cell lung cancer. J Thorac Cardiovasc Surg 2019;158:269-76.

41. Forde PM, Chaft JE, Smith KN, et al. Neoadjuvant PD-1 Blockade in Resectable Lung Cancer. N Engl J Med 2018;378:1976-86.

42. Rothschild SI, Zippelius A, Prince SS, ET AL. SAKK 16/14: Anti-PD-L1 antibody durvalumab in addition to neoadjuvant chemotherapy in patients with stage IIIA(N2) non-small cell lung cancer (NSCLC) - A multicenter single-arm phase II trial. J Clin Oncol 2020;38:9016.

43. Ozdemir S, Finkelstein EA. Cognitive Bias: The Downside of Shared Decision Making. JCO Clin Cancer Inform 2018;2:1-10.

44. McAlpine K, Lewis KB, Trevena LJ, et al. What Is the Effectiveness of Patient Decision Aids for Cancer-Related Decisions? A Systematic Review Subanalysis. JCO Clin Cancer Inform 2018;2:1-13.

45. Ankolekar A, Dekker A, Fijten R, et al. The Benefits and Challenges of Using Patient Decision Aids to Support Shared Decision Making in Health Care. JCO Clin Cancer Inform 2018;2:1-10.

46. Treffers T, Putora PM. Emotions as Social Information in Shared Decision-Making in Oncology. Oncology 2020;98:430-7.

47. Iseli T, Berghmans T, Glatzer M, et al. Adverse events reporting in stage III NSCLC trials investigating surgery and radiotherapy. ERJ Open Res 2020;6:00010-2020. 